Ciência Florestal, Santa Maria, v. 4, n. 1, p. 159-167, 1994

ISSN 0103-9954

\title{
METODOLOGIA ALTERNATIVA PARA O TRATAMENTO DE EFLUENTES DE INDÚSTRIA DE CELULOSE KRAFT
}

\section{ALTERNATIVE METHODOLOGY FOR TREATMENT OF THE EFFLUENTS FROM CELULOSE KRAFT INDUSTRY}

\section{Sonia Maria Bitencourt Frizzo ${ }^{1}$ Maria Cládis Mezzomo da Silva ${ }^{2}$ Ayrton Figueredo Martins $^{3}$ Celso Edmundo Foelkel ${ }^{4}$}

\section{RESUMO}

São descritos procedimentos para o tratamento terciário dos efluentes da indústria Riocell S.A. (Guaíba, RS), com base no reaproveitamento de resíduos sólidos e de excedentes de processo químico. As amostras de efluente foram caracterizadas em relação a sua cor aparente a a seu teor em organohalogenados sendo após, submetidas a polimento com dois coagulantes/ floculantes distintos, utilizando resíduos sólidos de processo ("dreg", "grit", cinzas) e carvão ativo como coadjuvantes. Uma apreciável redução na cor aparente e no teor de organo-halogenados dos efluentes finais foi observada, bem como uma economia no gasto com coagulantes/floculantes.

Palavras-chave: processos químicos; efluentes; celulose.

1 Engenheira Florestal, Mestranda, Professora Adjunta do Departamento de Química, Centro de Ciências Naturais e Exatas, Universidade Federal de Santa Maria, Av. Roraima, 1000, CEP 97105-900, Santa Maria (RS), Brasil.

2 Engenheira Florestal, MSc., Professora Titular do Departamento de Química, Centro de Ciências Naturais e Exatas, Universidade Federal de Santa Maria, Av. Roraima, 1000, CEP 97105-900, Santa Maria (RS), Brasil.

3 Químico/Químico Industrial, Dr., Professor Titular do Departamento de Química, Centro de Ciências Naturais e Exatas, Universidade Federal de Santa Maria, Av. Roraima, 1000, CEP 97105-900, Santa Maria (RS), Brasil.

4 Engenheiro Agrônomo, MSc., Professor do Programa de Pós-graduação em Engenharia Florestal, Centro de Ciências Rurais, Universidade Federal de Santa Maria, Av. Roraima, 1000, CEP 97105-900, Santa Maria (RS), Brasil. 


\begin{abstract}
Procedures based on the surplus of chemicals for the tertiary treatment process of the Riocell S.A. (Guaíba, RS state - Brazil), are described. The effluent samples were characterized in relation to their colors and contents of organ-halogenated compounds and then submitted to a conditioning step with application of two different flocculants using solid residues of process ("dreg", "grit", ashes) and active carbon as adjuncts. An appreciable reduction of the color and content in organ-halogenated compounds was observed, as well as an economy on costs with flocculants.
\end{abstract}

Keywords: chemicals process; effluent; cellulose.

\title{
INTRODUÇÃO
}

A possibilidade de utilização de resíduos sólidos ("dreg", "grit" e cinzas), no tratamento de efluentes, e, de excedente (cloro), na produção de cloreto férrico, ambos sub produtos da linha de produção, constitui uma elegante alternativa para o reaproveitamento de resíduos. $\mathrm{O}$ uso de carvão ativado como coadjuvante de floculação, ademais, complementa estudos preliminares (AWWA, 1974, CHEREMISINOFF \& MORRESI, 1980, FROEHLICH, 1987 e GRULICH et al., 1973).

O estudo da concentração de organo-halogenados reunidos (AOX) faz-se necessário, outrossim, em virtude do uso de cloro e dióxido de clore em um das estágios do branqueamento da polpa (MARWAH et al., 1981 e SUSS et al., 1991). Em contato com a lignina, este produto, além de atribuírem a cor água, geram compostos organo-halogenados, que, quando não controlados, podem contaminar a estrutura trófica (CELESTE \& CÁCERES, 1987).

\section{MATERIAL E MÉTODOS}

\section{Amostragem}

As amostras de efluente, sem que tenham recebido qualquer tipo de coagulante, foram coletadas do transbordo do tratamento secundário da Riocell S.A., tendo sido acondicionadas em recipientes de vidro, 
descontaminados, e armazenados em câmera fria (AGUDO, 1987, DIN, 1985 e STANDARD METHODS FOR THE EXAMINATION OF WATER AND WASTEWATER, 1985).

\section{Desenvolvimento do Trabalho}

O presente estudo dividiu-se em três estágios: caracterização geral do efluente, (verificação da cor aparente e da concentração de AOX); aplicação das tratamentos com coagulantes $\{\mathrm{Al} 2(\mathrm{SO} 4) 3$ e $\mathrm{FeCl} 3\}$ e adsorventes (carvão ativo 1 e 2, "dreg", "grit" e cinza pesada); caracterização do afluente após os tratamentos (determinação de cor aparente e de AOX).

"Dreg" e "grit" são resíduos sólidos gerados durante a fase de recuperação do licor de cozimento da produção de polpa sulfato. Cinza pesada provém da caldeira de força, da queima de carvão mineral.

\section{Métodos de Análise}

A cor aparente foi medida segundo o método de análise fotométrico, com auxílio do aparelho Micronal B295, com comprimento de onda de 440nm; a curva analítica foi obtida com solução padrão Pt-cloreto cobaltoso (STANDARD METHODS FOR THE EXAMINATION OF WATER AND WASTEWATER, 1985). A determinação de organo-halogenados totais (AOX) foirealizada comauxílio do aparelho de adsorção marcaEUROGLASECS 1000 (DIN, 1985 e SCAN, 1989).

Os tratamentos aplicados ao afluente constaram de 12 combinações entre coagulantes/floculantes e adsorventes: $\mathrm{FeCl} 3$ sem adsorventes; $\mathrm{FeCl} 3 \mathrm{e}$ carvão 1; $\mathrm{FeCl} 3$ e carvão 2; $\mathrm{FeCl} 3$ e "grit"; $\mathrm{FeCl} 3$ e "dreg"; $\mathrm{FeCl} 3$ e cinza; $\mathrm{Al}(\mathrm{SO} 4) 3$, sem adsorventes; $\mathrm{Al}(\mathrm{SO} 4) 3$ e carvão 1; $\mathrm{Al}(\mathrm{SO} 4) 3$ e carvão 2; $\mathrm{Al}(\mathrm{SO} 4) 3$ e "grit"; $\mathrm{Al}(\mathrm{SO} 4) 3$ e "dreg"; $\mathrm{Al}(\mathrm{SO} 4) 3$ e cinza.

Esta etapa foi realizada com o auxílio do teste de Jarro, onde foram colocados, respectivamente, o adsorvente, a amostra e o coagulante, corrigindo-se o pH para 4,7 (no caso de $\mathrm{Al}(\mathrm{SO} 4) 3$ ) e 4,0 (para $\mathrm{FeCl} 3$ ). O programa de agitação foi: alta velocidade, por 1 minuto, seguindo-se 15 minutos, à baixa velocidade; depois disso, deixou-se em repouso por 30 minutos. 
No sobrenadante resultante, determinou-se valores para a cor aparente e para o teor de AOX, de acordo com as normas mencionadas.

\section{RESULTADOS E DISCUSSÃO}

\section{Cor aparente}

A média da cor aparente do efluente, antes dos tratamentos foi de 1081,4 mg Pt.Co/l.

Baixos valores de cor, caracterizam os tratamentos considerados como os melhores (BLAKE \& ZUNCICH, 1991). Por esta razão, são aqui representados somente os resultados referentes as grupo de tratamentos que leva os menores valores de cor (mg Pt.Co/l.).

A Tabela 1 apresenta uma descrição estatística do comportamento da cor aparente do efluente quendo submetido aos tratamentos com os coagulantes/floculantes e adsorventes.

Obteve-se o valor de 12,54, com correção de Yates, no teste referente a classificação (maior cor aparente e menor cor aparente) x floculante (sulfato de alumínio e cloreto férrico). Constatando-se então que a classificação encontra-se associada com o tipo de floculante empregado; ou que a proporção

TABELA 1: Resumo estatístico do comportamento da cor aparente do afluente de Riocell, em função dos valores obtidos nas amostras submetidas aos tratamentos.

TABLE 1: Statistical summary of the apparent color of the behavior of affluent of Riocell company, depending on the values obtained for samples submitted to treatments.

\begin{tabular}{lclc}
\hline \multicolumn{1}{c}{ Medida Estatística } & $\begin{array}{c}\text { Cor Aparente } \\
(\mathrm{mgPt} . \mathrm{Co} / \mathrm{l})\end{array}$ & Medida Estatística & $\begin{array}{c}\text { Cor Aparente } \\
(\mathrm{mgPt} . \mathrm{Co} / \mathrm{l})\end{array}$ \\
\hline Número de observações & 258 & Terceiro quartil & 108,10 \\
Cor aparente & 84,73 & Amplitude interquartílica & 47,10 \\
Desvio padrão & 28,69 & Cor aparente modal & 108,10 \\
CVP (\%) & 33,85 & Menor valor encontrado & 33,00 \\
Primeiro quartil & 60,40 & Maior valor encontrado & 116,10 \\
Cor aparente mediana & 94,40 & Amplitude total & 83,10 \\
\hline
\end{tabular}

Ci. Fl., v. 4, n. 1, 1994 
de amostras tratadas com sulfato de alumínio, classificadas em menor cor aparente, é significativamente, diferente da proporção de amostras tratadas com cloreto férrico. A Tabela 2 ilustra a distribuição relativa das amostras.

A mesma estrutura de apresentação dos resultados foi feita para as diferentes concentrações dos coagulantes, bem como para os diferentes tipos de adsorventes e suas respectivas concentrações.

De acordo com Tabela 2, o sulfato de alumínio é o coagulante que apresentou os melhores resultados para a redução da com aparente, isto porque $83,33 \%$ das amostras foram agrupadas nos menores valores de cor aparente. A concentração de $400 \mathrm{mg} / \mathrm{l}$ de sulfato de alumínio foi a que agrupou um maior número de amostras com menores valores de cor aparente.

Todos os adsorventes podem ser usados, porque não apresentam diferenças significativas entre os diferentes tratamentos, com exceção do "grit". Economicamente, é mais atraente o uso de resíduos sólidos produzidos pela indústria, no casa "dreg" e cinza pesada, as maiores concentrações utilizadas, respectivamente 82,4 e $105,0 \mathrm{mg} / \mathrm{l}$.

TABELA 2: Distribuição relativa de amostras classificadas segundo o tipo de floculante e a classificação das amostras em função da cor aparente (mg Pt.Co/l.).

TABLE 2: Relative distribution of samples classified according to the type of flocculant and classification of samples depending on the apparent color (Pt.Co/l mg.).

\begin{tabular}{lcc}
\hline \multicolumn{1}{c}{ Classificação } & $\mathrm{A} 12(\mathrm{SO} 4) 3$ & $\mathrm{FeCl3}$ \\
\hline $\begin{array}{l}\text { Proporção de amostras classificadas no grupo de maior cor } \\
\text { aparente }\end{array}$ & 16,67 & 34,29 \\
$\begin{array}{l}\text { Proporção de amostras classificadas no grupo de menor cor } \\
\text { aparente }\end{array}$ & 83,33 & 65,71 \\
\begin{tabular}{l} 
Total \\
\hline
\end{tabular} & 100,00 & 100,00 \\
\hline
\end{tabular}

\section{AOX}

A média da concentração de AOX para o efluente sem tratamento foi de $3,55 \mathrm{mg} / \mathrm{l}$.

A Tabela 3 apresenta o comportamento do efluente obtido através do 
teste de Kruskal-Wallis (grupo que apresentou o menor valor para a média de AOX), em relação aos tratamentos a que foi submetido.

A Tabela 4 e a Figura 1 mostraram a percentagem de amostras classificadas com menor concentração de AOX, de acordo com os tipos de floculantes utilizados.

De acordo com a Tabela 4, o coagulante que apresentou melhor resultado foi o cloreto férrico, porque $88,57 \%$ das amostras foram classificadas como as que apresentaram menor concentração de AOX. Partindo-se deste fato, averiguou-se qual seria, então, a concentração mais adequada deste coagulante. Realizou-se, pois, da mesma forma que antes, o agrupamento do número de amostras com as concentrações de 200, 250 e $350 \mathrm{mg} / 1$, para as que tinham maior concentração em AOX e, também, para as que tinham menor concentração. Maior número de amostras com menor concentração de AOX, foram obtidas com as amostras tratadas com 359 ppm de cloreto férrico.

Frente ao exposto, foi necessário categorizar os dados em duas

TABELA 3: Resumo estatístico do comportamento do AOX do efluente, em função dos tratamentos.

TABLE 3: Statistical summary of the AOX effluent behavior in the treatments.

\begin{tabular}{lc}
\hline \multicolumn{1}{c}{ Medida Estatística } & AOX $(\mathrm{mg} / \mathrm{l})$ \\
\hline Número de observações & 88 \\
AOX média & 0,99 \\
Desvio padrão & 0,36 \\
CVP (\%) & 36,36 \\
Primeiro quartil & 0,68 \\
Cor aparente mediana & 0,97 \\
Terceiro quartil & 1,28 \\
Amplitude interquartílica & 0,60 \\
Cor aparente modal de AOX & 0,74 \\
Menor valor encontrado & 0,37 \\
Maior valor encontrado & 2,17 \\
Amplitude total & 1,80 \\
\hline
\end{tabular}

Ci. Fl., v. 4, n. 1, 1994 
TABELA 4: Distribuição relativa da amostras classificadas segundo o tipo de floculante e a classificação das amostras em função da concentração de $\mathrm{AOX}(\mathrm{mg} / \mathrm{l})$.

TABLE 4: Relative distribution of samples classified according to the type of flocculant and classification of samples according to the concentration of AOX (mg/1).

\begin{tabular}{lcr}
\hline \multicolumn{1}{c}{ Classificação } & $\mathrm{A} 12(\mathrm{SO} 4) 3$ & $\mathrm{FeCl3}$ \\
\hline $\begin{array}{l}\text { Número de amostras classificadas no grupo de maior concentração } \\
\text { de AOX }\end{array}$ & 45,83 & 11,43 \\
$\begin{array}{l}\text { Número de amostras classificadas no grupo de menor concentração } \\
\text { de AOX }\end{array}$ & 54,17 & 8,57 \\
TOTAL & 100,00 & 100,00 \\
\hline
\end{tabular}

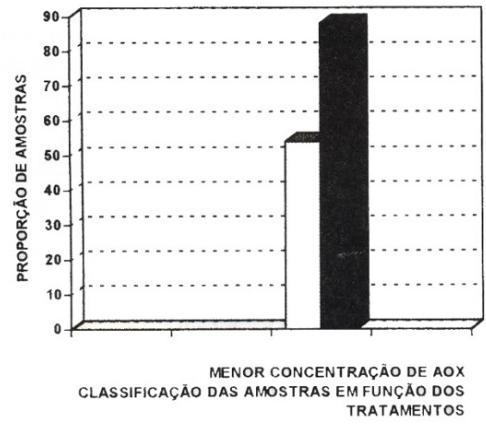

FIGURA 2: Distribuição relativa do número de amostras classificadas em função dos coagulantes/floculantes e da classificação correspondente a concentração de AOX (mg/l).

FIGURE 2: Relative distribution of the number of samples classified according to the coagulants/flocculants and the corresponding classification AOX concentration ( $\mathrm{mg} / \mathrm{l}$ ).

classes, sem adsorvente e com adsorvente. A indústria tem, naturalmente, interesse em aproveitar seus resíduos sólidos. Não há diferença significativa, ademais, entre os diversos tipos de adsorventes, segundo os vários procedimentos estatísticos empregados, com exceção do carvão 2 e do "grit", 
que apresentaram uma maior número de amostras com menores teores de AOX.

As concentrações testadas de carvão 2 (100, 150 e 200 mg/l) também não apresentaram diferenças significativas, bem como as concentrações de "grit" (58,2 e 78,3 mg/l).

\section{CONCLUSÕES}

Com base nos resultados apresentados, pode-se concluir que:

- o melhor coagulante/floculante para redução da cor aparente foi sulfato de alumínio na concentração de $400 \mathrm{mg} / \mathrm{l}$, coadjuvado pelos resíduos industriais "drg" (82,4mg/l) e cinza pesada $(105,0 \mathrm{mg} / \mathrm{l})$; o que é muito agradável, porque além de reduzir os resíduos sólidos, poderá promover uma economia em gastos com adsorventes;

- o melhor coagulante/floculante testado para redução de AOX foi o cloreto férrico na concentração de $350 \mathrm{mg} / \mathrm{l}$; certamente, um fato muito interessante, já que pode ser produzido na própria indústria, pelo aproveitamento de cloro excedente; o adsorvente mais efetivo na redução de AOX foi o carvão 2, que, por razões econômicas poderá ser usado na concentração de $100 \mathrm{mg} / \mathrm{l}$, seguindo-se do "grif" que deverá ser usado na maior concentração testada, ou seja, 78,3 mg/l;

- houve redução da cor aparente e o teor de AOX, no decurso dos diversos tratamentos propostos, o que evidencia a eficiência de todos os tratamentos investigados.

\section{REFERENCIAS BIBLIOGRÁFICAS}

AGUDO, E.G. Guia de coleta e preservação de amostras de água. São Paulo; CETESB, 1987. 150p.

AWWA Standards For Granular Activated Carbon. Journal American Water Works Association, Washington, v.66, n.11, p.672-681, nov. 1974.

BLAKE, N.R.; ZUNCICH, J.L. Reduction of chlorinated organic in pulp and paper mill effluent. In: ENVIRONMENTAL CONFERENCE, 1991, Atlanta. Anais... Atlanta: Tappi Press, 1991, v.1, p.445-451. 
BRYANT, C.W.; DETTMER, J.W.; BARKLEY, W.A. Removal of organic chlorine and color from kraft wastewater and lagoon sludge undernalternate treatment cinditions. In: ENVIRONMENTAL CONFERENCE, 1991, Atlanta. Anais... Atlanta: Tappi Press, 1991, v.2, p.807-812.

CELESTE, M.F.; CÁCERES, O. Resíduos de praguicidas clorados na represa do Ribeirão do Lobo (Broa) e nos seus rios tributários. Ciência e Cultura. São Paulo, v.39, n.1, p.66-70, jan. 1987.

CHEREMISINOFF, P.N.; MORRESI, A.C. Carbon adsorption applications. In: CHEREMISINOFF, P.N., ELLEBUCH, F. Carbon Adsorption handbook. Michigan: Ann Arbor, 1980. p.1-53.

DIN Determination of adsorbable organically bonded halogens (AOX): 38409. Germany. part. 14.

FROEHLICH, C. Melhoramento de efluentes da indústria de celulosa e papel por carvão biológico. Porto Alegre: UFRGS, 1987. 166p. Dissertação (Mestrado em Engenharia Florestal) - Universidade Federal do Rio Grande do Sul, 1987.

GRULICH, G. et al. Treatment of organic chemicals plant wastewater with the Dipontpact process. Alche Symposium Series, New York, v.69, n.129, p.127-134, 1973.

MARWAH, N.et al. A simple process modification to reduce chloroorganic (AOX) formation in the breachplant. In: ENVIRONMENTAL CONFERENCE, 1991, Atlanta. Anais... Atlanta: Tappi Press, 1991, v.2, p.1029-1031.

SCAN. Eflluents from pulp mills: organically bound chlorine by the AOX method. Denmark, 1989.

STANDARD methodsfor theexaminationof water and wastewater. Washington: APHA AWWA, 1985. 1268p.

SUSS, H.U.; NUMMERFROH, N.; EUL, L.W. Envirnmental aspects of short-sequence bleaching Atlanta: Jamell, Hasan, 1991. p.482-492. 